\title{
Bone metastases in neuroendocrine tumors
}

\author{
Zamborsky R ${ }^{1}$, Svec $\mathrm{A}^{2}$, Kokavec $\mathrm{M}^{1}$, Galbavy $\mathrm{S}^{3,4}$ \\ Department of Orthopaedics, Faculty of Medicine, Comenius University, Children's University Hospital, \\ Bratislava, Slovakia. radozamborsky@gmail.com
}

\begin{abstract}
Neuroendocrine tumors arise from various cells that form a part of the endocrine system and account for a small number of cases encountered by oncologists in clinical practice. The clinical incidence of these tumors used to be low, and newer imaging modalities have now begun to be used for detecting bone metastases at an earlier stage. Bone metastases arising from neuroendocrine tumors are a well-recognized complication. Their presence carries along a poor prognosis. Clinical symptoms are similar to those encountered in other forms of cancer that are complicated by bone metastasis. Over the last decade or so, the clinical detection, diagnostic methods and treatment strategies have changed dramatically, and new treatments are emerging slowly. The indolent course of neuroendocrine tumors and the development of bone metastasis have limited our current knowledge on how to best prevent and manage the condition. Current information available from clinical studies is marred by paucity and small sample sizes, making further clinical trials an absolute necessity. In this review, we discuss the current status in the diagnosis and management of bone metastases arising from neuroendocrine tumors (Fig. 3, Ref. 28). Text in PDF www.elis.sk.

KEY WORDS: endocrine tumors, metastatic tumor/carcinoma, neoplasm metastasis, bone metastasis, biomarkers, diagnostic imaging, bone resection.
\end{abstract}

\section{Introduction}

Neuroendocrine tumors (NET) are rare tumors arising from various cells forming a part of the endocrine system. They account for only $0.5 \%$ of all tumors and can present in a variety of ways (1). Unfortunately, there is a paucity of studies that have assessed metastases of these tumors. Neuroendocrine tumors can arise from the lung, liver, pancreas and intestine, with the latter being the most common site of origin. Numerous studies have looked at carcinoid tumors and its clinical effects, though there are case reports that have evaluated NETs of other origin.

Innately, neuroendocrine tumors have the property of metastasizing to the bone, liver, brain and lung. Bone metastasis, once thought to be a rare feature of neuroendocrine tumors, is now a well-recognized clinical complication requiring specific investigations and treatment, and is marred with poor long-term outcomes and prognoses (2). The impact it can have on the quality of life of the individual can be tremendous, and can lead to loss of mobility, pain and dependence on assistance for activities of daily living. It

${ }^{1}$ Department of Orthopaedics, Faculty of Medicine, Comenius University, Children's University Hospital, Bratislava, Slovakia, ${ }^{2}$ st Department of Orthopaedics and Traumatology, Faculty of Medicine, Comenius University and University Hospital, Bratislava, Slovakia, ${ }^{3}$ Department of Pathology, St. Elizabeth University of Health and Social Sciences and St. Elisabeth Institute of Oncology, Bratislava, Slovakia, and ${ }^{4}$ Institute of Forensic Medicine, Faculty of Medicine, Comenius University, Bratislava, Slovakia Address for correspondence: R. Zamborsky, MD, PhD, Department of Orthopaedics, Comenius University, Children's University Hospital Bratislava, Limbova 1, SK-833 40 Bratislava, Slovakia. Phone: +421.905363974

Acknowledgements: Supported with Grant KEGA 071UK-4/2016. also places a large demand on health care resources, amounting to high annual expenditure and contributing to considerable morbidity. In this review article, we take a look at common neuroendocrine tumors and management of bone metastases.

\section{Epidemiology}

In a study performed in France on 668 patients with neuroendocrine tumors, $6.4 \%$ of them were found to have bone metastases (3). Rates of up to $12 \%$ have been seen in carcinoid tumors, however various clinical studies have shown an incidence of up to $40 \%$. This inconsistency could be attributed to better diagnostic techniques that are currently available. Medullary carcinoma of the thyroid is a recognized etiology involving the bones.

In a study conducted by Van Loon et al, bone metastases were seen in $23 \%$ of pheochromocytomas and sympathetic paragangliomas, $9 \%$ of carcinoid tumors and $20 \%$ of high-grade NETs (4). Typically, the bone metastases seen with NET are osteosclerotic in nature and involve the axial skeleton, although around $10 \%$ can be osteolytic with around $15 \%$ to $30 \%$ involving the pelvis and femur (5). Foregut and hindgut primaries seem to be the main source of bone metastases, while midgut primaries are a rare etiology. However, it appears that in a number of cases there may be no preferential site (6).

Early detection of bone metastasis is essential given the poor prognosis and difficulties with surgical resection in such cases. The primary concern associated with bone metastasis is that it can sometimes be the first detected clinical feature of NETs. However, it is not uncommon for metastasis to occur years after the diagnosis of NETs themselves, with recent studies showing a median 

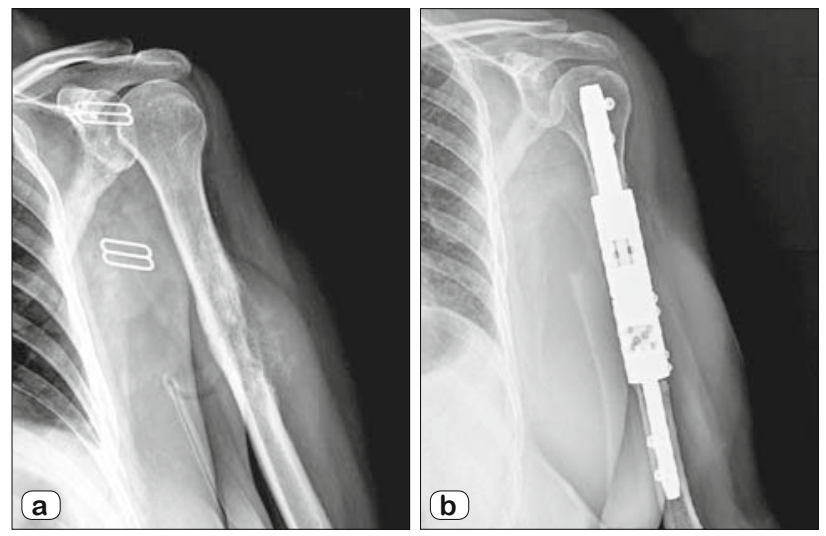

Fig. 1. a) Adrenal cortex (functioning) metastatic bone lesion of the left humerus in a 58-year-old woman presenting with Cushing's syndrome, b) postoperative $X$-rays after en-block resection of the humeral shaft for metastatic bone lesion and reconstruction with modular intercalar prosthesis.

time of detection of 41.6 months to detection of bone metastasis (4). This makes the prediction of complication a difficult task (1).

\section{Mechanism and site of bone metastasis}

The mechanism of bone metastasis in neuroendocrine tumors is similar to those seen in other forms of cancer. Bones appear to be a preferential site due to high vascularity of the bone marrow. Cancer cells produce adhesion factors that allow them to bind to cells in the marrow. The further release of growth factors promotes cancer cell proliferation, thus allowing increased growth of the tumor within the bone.

Other pathophysiological mechanisms have been postulated in the mechanism of bone metastasis. For example, cancer cells arising from pheochromocytomas and sympathetic paragangliomas may spread to the skeleton due to a homing mechanism that is dictated through expression of CXCR4 and CCR9 (7).

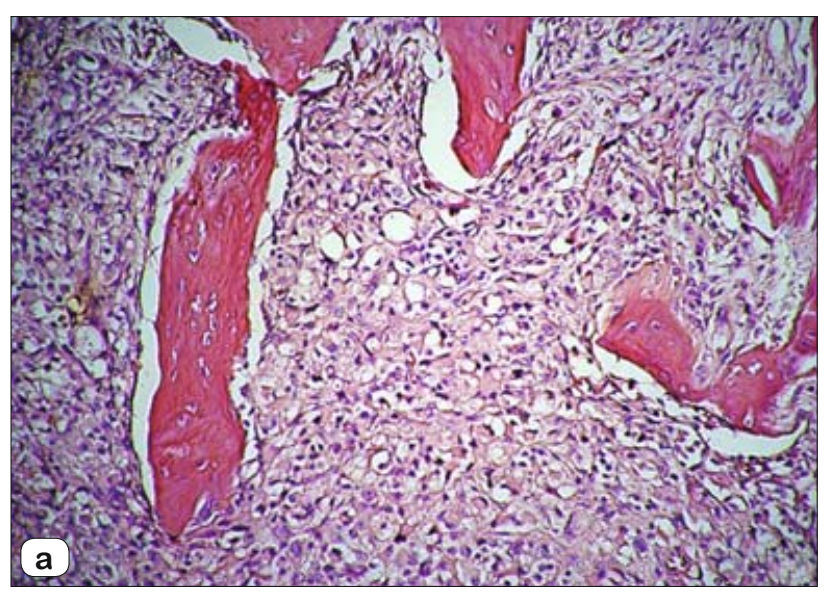

Bone metastasis varies depending on the site of the primary tumor, and the rate of skeletal involvement appears to be higher with pulmonary carcinoid tumors as compared to those arising from the gastrointestinal tract.

The axial skeleton is the most common site of metastasis from carcinoid tumors. The axial skeleton is rich in red marrow, and its high vascularity and abundance of cells make it the ideal environment for metastasis to occur. Nearly $40 \%$ of the spread involves the thoracic vertebra, while the distribution of metastases is similar to those of non-carcinoid tumors (8). Lumbar vertebra spread is seen in $34 \%$, cervical vertebra spread in $32 \%$ and a small proportion involves the ribs and pelvis (9).

Neuroendocrine tumors such as carcinoid tumors have a slow and indolent course, and bone metastases may only occur years after the initial diagnosis. However, this does not appear to be a hard and fast rule. Adrenocortical carcinoma is a very rare tumor with a poor prognosis. About half of them are hormone-secreting tumors and can present with skeletal metastases in a postmenopausal woman (Figs 1-3).

\section{Clinical symptoms in metastatic involvement of the bone}

Skeleton-related events, or SREs, often present after the discovery of bone metastasis, though in some cases it may pre-

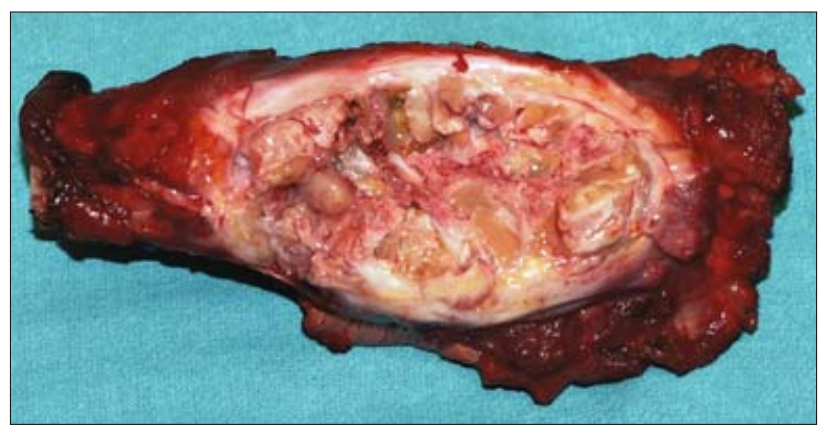

Fig. 2. En-block resection of skeletal metastasis (adrenocortical carcinoma).

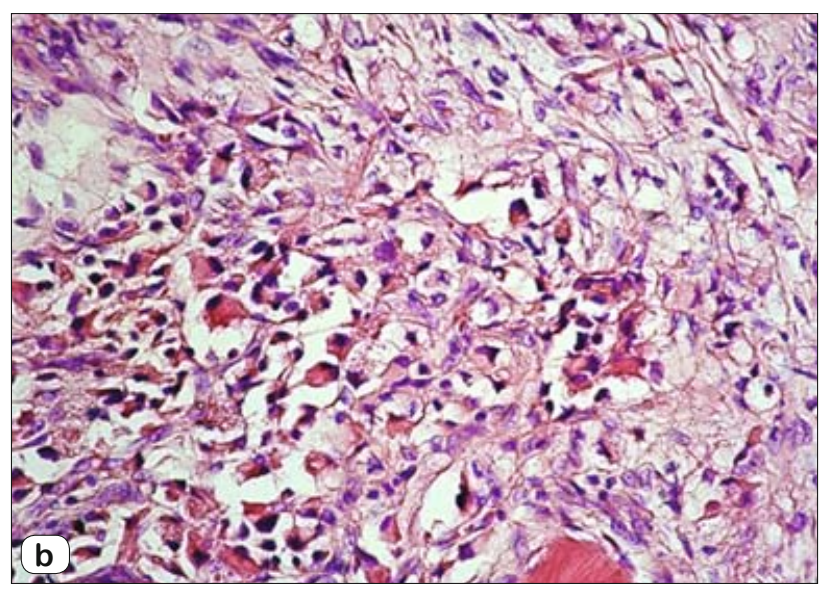

Fig. 3. a) a bone metastasis from an adrenocortical carcinoma. Irregularly arranged tumor cells between bony trabeculae, showing marked atypia and hyperchromasia $(\mathrm{H}$ and $\mathrm{E}, \mathbf{x 1 0 0})$, b) the tumor cells have abundant eosinophilic cytoplasm and enlarged hyperchromatic nuclei with prominent nucleoli. Numerous mitotic figures were also present (H and $\mathbf{E}$ x200). 
cede the diagnosis of metastatic lesions. The development of one SRE usually leads to the development of further SREs. In a study assessing 137 patients with malignant pheochromocytomas and sympathetic paragangliomas, the development of a second SRE was noticed within 10 months of development of the first event. Bearing a similarity to breast cancer, the authors also found the median time of 4.4 months to development of the first SRE (10).

\section{Pain}

The primary symptom that is encountered in bone metastasis is pain, which is often disproportionate to the degree of metastases. Numerous pathophysiological mechanisms for pain have been postulated, including increased production of cytokines, osteolysis and nerve infiltration by cancerous lesions (11). Mechanical back pain may occur due to spinal instability.

In severe cases, the pain can be debilitating, but a large proportion of patients do not experience pain at all. In patients with bone metastasis from carcinoid syndrome, only $4 \%$ of cases were clinically detected in advanced cases (12), while $42 \%$ were detected post mortem (13). In a retrospective study looking at 128 patients with malignant pheochromocytoma and sympathetic paraganglioma, severe pain was the most common skeleton-related event (SRE), though only 67 subjects had at least 1 SRE (10).

However, other studies have demonstrated a higher incidence of pain (4). This discrepancy could potentially lead to under-reporting of bone metastases (6), and subsequently to a significant reduction in the number of patients seeking treatment for bone metastases.

\section{Pathological fractures}

Pathological fractures are another clinical feature recognized as the root cause of pain in most patients. The destruction of bone tissue by the infiltrating malignant lesion results in the loss of weight bearing capacity of the bones. Early stages see the formation of micro-fractures, which are the primary cause of pain. However, the pain in this stage is rarely debilitating, and advanced cancers that extend into the epidural space can significantly limit mobility and impair the quality of life.

With respect to NETs, Loon et al reported only $9 \%$ of their study subjects suffering a pathological fracture (4), with similar observations being reported by other groups (6).

\section{Spinal cord compression}

This is one of the most dreaded complications, and requires emergency management. Spinal cord compression can cause pain that gets worse at night, and is often localized with nerve fibers in close proximity to the metastatic lesions. Radicular pain may present in the initial stages, which can then progress to complex neurological symptoms. Advanced cases of spinal nerve compression can lead to urinary retention or incontinence associated with paralysis.

Symptoms related to spinal column compression are seen in $10 \%$ of patients (4). Spinal cord compression from neuroendocrine tumors often involves the thoracic and lumbar spine, though there are a handful of reports suggesting cervical involvement (14). Cauda equina syndrome is a major concern requiring emergency treatment through decompression and possible use of radiotherapy.

\section{Hypercalcemia}

Hypercalcemia in bone metastasis related to increased bone turnover secondary to the release of a number of different paracrine and humoral factors by the tumor cells. This phenomenon may be accompanied by elevation in levels of circulating parathyroid hormone-related peptide levels.

However, hypercalcemia is rarely present in bone metastases secondary to neuroendocrine tumors, and has only been reported up to $4 \%(4,6)$. A significantly higher incidence of hypercalcemia is seen in metastases arising from pheochromocytoma and sympathetic paragangliomas as compared to carcinoid, pancreatic neuroendocrine tumor and high-grade neuroendocrine carcinoma (4).

The other symptoms that are encountered are a consequence of the above, and include limited mobility, psychological distress and inability to perform day-to-day activities.

\section{Diagnosis}

The diagnosis of bone metastases from NETs is quite complex, and often requires a combination of different modalities before the treatment options are discussed. Most of the laboratory investigations that are performed remain non-specific to bone metastases arising from neuroendocrine tumors, though their applicability in reaching and confirming the diagnosis still remains valid.

Prior to making the diagnosis of bone metastases, the confirmation of the presence of a neuroendocrine tumor is essential. Once this has been done, subsequent laboratory and radiological investigations can be chosen as appropriate.

\section{Laboratory studies}

Laboratory results are commonly based around the presence or absence of specific markers in the blood. These markers are helpful in determining the primary etiology of the metastases.

\section{Chromogranin A ( $\mathrm{Cg} A)$}

This acidic glycoprotein is composed of 439 amino acids and is an integral part of the neuroendocrine cells. Serum detection or tissue analysis can estimate levels of elevated chromogranin A levels, which is a non-specific marker of neuroendocrine tumors. Elevated levels can be seen in carcinoid tumors, pheochromocytomas, VIPomas, glucagonomas, gastrinomas and insulinomas, along with medullary thyroid carcinomas as well.

Studies have demonstrated that elevated circulating levels of $\mathrm{CgA}$ possess a high sensitivity and specificity of up to $81 \%$ (15). However, there do not appear to be significant differences in the levels based on the location of the primary tumor. Higher levels of this marker are seen in individuals who have metastatic neuroendocrine tumors (15). 


\section{Bone-specific Alkaline Phosphatase (BSAP)}

This is also a non-specific marker for bone metastases originating from a NET. As a more specific marker of bone metastases, BSAP levels detect the degree of osteoblastic activity i.e. bone formation. BSAP levels are considered a marker of adverse prognosis, with research suggesting superiority over chromogranin A (16). However, BSAP does not have to play a role in distinguishing patients with and without bone metastases (6).

There are other bone markers that may play a role in the diagnosis of metastatic bone lesions from NETs. Serum aminoterminal propeptide of type I collagen and serum cross linked amino-terminal telopeptide of type I collagen (NTx) are useful indicators of osteoblastic and osteoclastic activity, respectively. Once again, the presence of these markers is not confirmative of bone metastasis (6).

\section{Imaging studies}

The primary purpose of imaging studies is not just to determine the degree of anatomical bone involvement, but also to determine the functional aspects of bone metastases. However, conventional radiographic studies can occasionally miss the presence of metastatic lesions (17).

In order to determine the extent of metastasis, more than one imaging test is usually needed. Head to head studies have been conducted assessing the sensitivity and specificity of somatostatin receptor scintigraphy against that of a CT scan and conventional ${ }^{99 \mathrm{~m}} \mathrm{Tc}$-biphosphonates bone scintigraphy. Traditional bone scintigraphy still remains a popular option, but is limited by its dependence on the level of osteoblastic activity within the bone and blood supply to the bone itself(18). Furthermore, scintigraphy using ${ }^{123}$ I-MIBG can underestimate the extent of bone metastases, primarily due to the associated uptake by nearby vital structures such as the liver and kidney.

\section{Octreotide scintigraphy}

Also called somatostatin receptor scintigraphy (SRS), this procedure is a useful diagnostic test in the diagnosis of bone metastasis. The conducted studies have shown this to be as effective as conventional bone scintigraphy. However, the reported rates of skeletal metastasis vary, with the highest being around $70 \%$ in patients with metastatic carcinoid syndrome (6). This low rate could be related to underestimation of bone metastases using this form of scintigraphy.

Despite its lower sensitivity, octreotide scintigraphy is still considered a good first step in the diagnosis of bone metastasis. Newer methods of diagnosis are emerging, and the novel somatostatin analogue ${ }^{68} \mathrm{Ga}-1,4,7,10$-tetraazacyclododecane- $N, N^{\prime}, N^{\prime \prime}, N^{\prime \prime \prime}$-tetraacetic acid-d-Phe ${ }^{1}$-Tyr ${ }^{3}$-octreotide $\left({ }^{68} \mathrm{Ga}\right.$-DOTATOC) uptake using positron emission tomography has demonstrated benefits and superiority to conventional SRS, bone scintigraphy and CT imaging (19).

\section{Magnetic resonance imaging}

Magnetic resonance imaging is regarded as the most sensitive and accurate test in the diagnosis of metastatic bone lesions arising from NETs (20). It possesses the capability of detecting early bone marrow deposits due to high spatial resolution, making it a useful tool in the evaluation of bone metastases (21). This feature also makes it a useful test to monitor the progress following the commencement of treatment.

In a study assessing the sensitivity of investigation modalities in the diagnosis of bone metastasis, Meijer et al found MRI to be $100 \%$ sensitive as compared to $70 \%$ for octreotide scintigraphy and $90 \%$ for conventional bone scintigraphy (6). However, these numbers vary and small studies have demonstrated a sensitivity ranging from $90 \%$ to $100 \%$. Similar sensitivities have been demonstrated in the diagnosis of bone metastases from noncarcinoid tumors.

\section{Recommendation}

The available evidence by comparing different imaging modalities is poor, and more studies need to be performed before a clearcut protocol is available to detect the extent of bone metastases. While MRI bears the advantage of higher sensitivity, concerns have been raised about its limited visualization capabilities, requiring multiple images and sittings. Some groups recommend the use of a combination of modalities, starting with octreotide scintigraphy followed by MRI if appropriate (6). The 2012 ENETS consensus guidelines recommend using PET/CT with $68 \mathrm{Ga}-\mathrm{SSA}$ such as ${ }^{68} \mathrm{Ga}$-DOTATOC as a way to detect early bone metastasis (22).

\section{Plain radiography}

Unless the lesion is large and visible enough, this modality has a very low sensitivity of detecting bone metastasis (6), and only remains useful as a screening test. If the clinical suspicion is high, bone scintigraphy (either conventional or octreotide) has a better sensitivity.

\section{Histopathology}

Performing a histo-pathological diagnosis is unlikely to be useful given the higher sensitivity of non-invasive imaging available. Routine performance is not recommended.

\section{Treatment}

Bone metastases have a prognostic significance, and various available treatment modalities are aimed towards extending the survival and improving the quality of life. Primary treatment modalities must be targeted towards the cause of bone metastases.

\section{Analgesia}

Pain management is of prime importance in bone metastasis. Non-steroidal anti-inflammatory drugs are a useful starting treatment, though advanced cases and those with significant pain may require radiotherapy. Palliative radiotherapy in doses of 8 Gy appear to be superior to $4 \mathrm{~Gy}$, though the ideal dose for adequate pain control is still questioned (23).

\section{Bisphosphonates}

The role of bisphosphonates has not been clearly established in the management of NET-related bone metastasis. However, they 
do appear to have benefits in the management of metastasis-related pain and quality of life. Therefore, their clinical use as a palliative treatment option is relevant and may be decided on a case-by-case basis. Most of the current evidence relates to bone metastasis associated with lung carcinoma and other related cancers, and there is a paucity of trials assessing NET-related bone metastasis owing to the rarity of the condition itself. More studies are necessary to define the exact role of bisphosphonates in treating metastasis related to NETs.

The study of the receptor activator of nuclear factor- $\kappa \mathrm{B}$ (RANK) has been assessed as well, and as is the case with bisphosphonates, further clinical studies are required to assess its clinical applicability.

In both the above treatments, the duration of therapy and the dosage is yet to be clearly established.

\section{Chemotherapy}

Chemotherapy options depend on the grade of the tumor and are often not dependent on the site of metastasis. The use of Peptide Receptor Radionuclide Therapy (PPRT) has been shown to be effective in the management of bone metastases accompanying NETs in some studies. Clinical effects using either ${ }^{177} \mathrm{Lu}$ or ${ }^{90} \mathrm{Y}$ have demonstrated a positive response when assessed through somatostatin receptor scintigraphy. However, the treatment only appears to be palliative, with symptom control achieved and survival improved. The number of cycles of therapy deemed appropriate is yet to be established as is the dosing and the timing of treatment.

\section{Somatostatin analogues}

Somatostatin analogues along with the use of interferon therapy have been shown to have some effect in the management of NETs of gastro-enteropancreatic origin. Clinical studies have shown that octreotide, a somatostatin analogue that effectively binds to somatostatin type 2 and type 5 receptors, is useful in the management of neuroendocrine tumors. We have already discussed the clinical use of somatostatin receptor scintigraphy in the detection and diagnosis of NETs, and the same principle is utilized in treating bone metastasis as well.

Indium ${ }^{111}$ has been used in conjunction with somatostatin analogues in the treatment of metastatic NETs. As a gamma emitter, it emits both gamma rays and conversion electrons, allowing it to be internalized into the metastatic cells. ${ }^{111}$ In-pentreotide has been used by a few authors with some success in managing bone metastasis. In a case report published by van der Heil et al, using ${ }^{111}$ In-penteotride over a period of 11 months at $6 \mathrm{GBq}$ per dose showed some regression of disease as evidenced by scintigraphy (24). Biomarkers such as Chromogranin A showed no significant increase during the course of the treatment.

Yttrium-labeled somatostatin analogues have been used by some authors with some advantages over Indium ${ }^{111}$. However, follow up of treatment to assess clinical effects may be difficult, and irradiation-associated side effects may be more prominent in comparison (24). Clinical trials assessing the benefits of ${ }^{90} \mathrm{Y}$ DOTATOC had shown promising results, but continued treatment led to the development of renal failure (25).
In a study assessing the effects of somatostatin analogue ${ }^{177} \mathrm{Lu}-$ octreotate, a reduction in the size of the GEP tumor was noticed in $47 \%$ of patients. There were no pituitary side effects reported as the dosage used in the trial was low (26).

\section{${ }^{131}$ I-metaiodobenzylguanidine (MIBG) therapy}

${ }^{131}$ I-metaiodobenzylguanidine (MIBG) therapy is a recognized palliative treatment in the management of metastatic NETs. Reduced levels of serum chromogranin A and 24-hour urinary 5HIAA have been reported in clinical trials, though its actual impact on long-term prognosis remains debatable. However, symptomatic benefit is seen in more than $50 \%$ of patients, bearing in mind the use of only a small dose of ${ }^{131}$ I-MIBG. Incidentally, the toxicity-related side effects were seen in nearly $30 \%$ of the study population, with bone marrow suppression being high up on the list, albeit mild (27).

Both ${ }^{111}$ In-pentreotide and ${ }^{131}$ I-MIBG appear to underestimate the presence of bone metastases, which could explain the variable incidence reported in different clinical studies (17).

\section{Radiotherapy}

As previously discussed, radiotherapy plays a role in pain management, and evidence suggests a role in palliative management of bone metastasis. Trials assessing dosing of radiotherapy have found 8 Gy delivered in a single fraction to be non-inferior to a higher dose of $20 \mathrm{~Gy}$ administered as multiple fractions (28). However, further studies that assess the true impact of radiotherapy are needed.

\section{Surgery}

The application of surgery as a treatment options for skeletal metastases arising from NETs is still limited. A more aggressive treatment approach may be appropriate for those patients in whom metastatic disease is limited to a solitary lesion at a single organ site. Extensive en-bloc resection with lymphadenectomy may be required in certain cases (Fig.1b). Excision is not recommended for multiple lesions, though solitary lesions and those leading to mechanical disability of the axial skeleton may benefit from a surgical approach.

\section{Prognosis}

The presence of bone metastasis with liver metastases is an indicator of poor prognosis, but data regarding actual survival rates are poor. From the available evidence, only around $50 \%$ of patients suffering from gastroenteropancreatic NEPs with metastasis survive beyond 5 years (28). Prognosis in advanced ACCs is very poor, with a 5 -year survival of $10 \%$ to $25 \%$ and a mean survival of 5 to 18 months. At the end of 6 months from the diagnosis, our patient is still alive with subjective improvement of her symptoms ( HYPERLINK $\backslash 1$ "Mig00" 29). Follow-up should be focused on monitoring tumor size and extension of metastases by CT scan and nuclear scanning.

\section{Conclusions}

Bone metastases are a well-recognized complication of neuroendocrine tumors, and may present late due to the indolent 
course of the primary tumor. Incidence rates vary due to this particular fact, and also due to the paucity of clinical trials that have specifically studied this aspect of NETs. Pain is the predominant symptom, and the treatment must be directed towards alleviating symptoms and improving quality of life. The low detection rate of bone metastases can delay treatments that can be offered to patients earlier on, and this delay is the likely cause for the poor prognosis associated with it. Research is still underway, and future trials will hopefully discover newer ways to detect bone metastases early, allowing for timely treatments and improved prognosis.

\section{References}

1. Visser BG, Taal O. Epidemiology of neuroendocrine tumours. Neuroendocrinology 2004; 80 (Suppl 1): 3-7.

2. Dawson E, Haskell CM, Batzdorf U Kirkpatrick DB. Metastatic carcinoid presenting as a spinal tumor. Surg Neurol 1975; 4: 283-287.

3. Mitry C, O'Toole E, Louvet D, Pillon C, Cadiot D, Borson-Chazot G, Aparicio F, Ducreux T, Lecomte M, Etienne T, Lombard-Bohas PL. Thirteen-month registration of patients with gastroenteropancreatic endocrine tumours in France. Neuroendocrinology 2009; 89 (2): 217-222.

4. Zhang L, Keiser J, Carrasco C, Glass K, Ramirez MT, Bobiak S, Nakakura EK, Venook AP, Shah MH, Bergsland EK Van Loon K. Bone metastases and skeletal-related events from neuroendocrine tumors. Endocrine Connections 2015; 4 (1): 9-17.

5. Gibril F, Doppman JL, Reynolds JC, Chen CC, Sutliff VE, Yu F, Serrano J, Venzon DJ, Jensen RT. Bone metastases in patients with gastrinomas: a prospective study of bone scanning, somatostatin receptor scanning, and magnetic resonance image in their detection, frequency, location, and effect of their detection on management. J Clin Oncol 1998; 16 (3): 1040-1053.

6. Wim G, van der Veer E, Jager PL, van der Jagt EJ, Piers BA, Kema IP, de Vries EGE, Pax HB, Meijer W. Bone metastases in carcinoid tumors: clinical features, imaging characteristics, and markers of bone metabolism. J Nucl Med 2003; 44 (2): 184-191.

7. Ross PL, Wright KN, Hayashida ME, Santagata CY, Barontini S, Kung M, Sanso AL, Powers G, Tischler JF, HodincAS, Dahia R. A HIF1alpha regulatory loop links hypoxia and mitochondrial signals in pheochromocytomas. PLoS Genet 2005; 1 (1): 72-80.

8. Tubiana-Hulin M. Incidence, prevalence and distribution of bone metastases. Bone 1991; 12 (Suppl): 9-10.

9. Takeshi, Taketoshi Yasuda, Kayo Suzuki, Masahiko Kanamori, Tomoatsu Kimura. Hori. Skeletal metastasis of carcinoid tumors: Two case reports and review of the literature. Oncol Lett 2012; 3 (5): 1105-1108.

10. Lynn Palmer MJ, Hofmann MC, de la Cruz M, Moon BS, Waguespack SG, Habra MA, Ayala-Ramirez CJ. Bone metastases and skeletalrelated events in patients with malignant pheochromocytoma and sympathetic paraganglioma. J Clin Endocrinol Metab 2013; 98 (4): 1492-1497.

11. Mercadante S. Malignant bone pain: pathophysiology and treatment. Pain 1997; 69 (1-2): 1-18.

12. Tiensuu E, Holmberg L, Stridsberg M, Eriksson B, Theodorsson E, Wilander EJ. Carcinoid tumors: analysis of prognostic factors and survival in 301 patients from a referral center. Ann Oncol 1997; 8 (7): 685-690.

13. William EM, Ross CR. The carcinoid syndrome: comparison of 21 necropsy subjects with carcinoid heart disease to 15 necropsy subjects without carcinoid heart disease. Amer J Med 1985; 79 (3): 339-354.
14. Nishikawa JAH, Jamous MA, Grahame-Smith Gray DG. Spinal cord compression due to carcinoid metastasis. Postgrad Med J 1988; 64 (755): 703-705.

15. Öberg B, Stridsberg K, Eriksson M. Tumor markers in neuroendocrine tumors. Digestion 2000; 62 (Suppl 1): 33-38 .

16. Thomas E, Sengupta TP, Paulus J, Fawzia A, Duh MS, Kulke Clancy HM. Alkaline phosphatase predicts survival in patients with metastatic neuroendocrine tumors. Digest Dis SCI 2006; 51 (5): 877-884.

17. Hoefnagel CA, Boot H, Valdes Olmos RA, Taal Zuetenhorst BG. Evaluation of 111In-pentetreotide, 131I-MIBG and bone scintigraphy in the detection and clinical management of bone metastases in carcinoid disease. Nucl Med Commun 2002; 23 (8): 735-741.

18. Frederick D, Fahey FH, Packard AB, Davis RT, Alavi A, Treves Grant ST. Skeletal PET with 18F-fluoride: applying new technology to an old tracer. J Nucl Med 2008; 49 (1): 68-78.

19. Gabriel DM, Henninger B, Kendler D, Uprimny C, Dobrozemsky G, Decristoforo C, Bale RJ, Jaschke W, Virgolini Putzer IJ. Bone metastases in patients with neuroendocrine tumor: 68Ga-DOTA-Tyr3octreotide PET in comparison to CT and bone scintigraphy. J Nucl Med 2009; 50 (8): 1214-1221.

20. Geoffroy MPO, Laissy JP, Lebtahi R, Silbermann-Hoffman O, Henry-Feugeas MC, Cadiot G, Mignon M, Schouman-Claeys Debray E. Imaging appearances of metastases from neuroendocrine tumours of the pancreas. Brit J Radiol 2001; 74 (887): 1065-1070.

21. Mills DCM, Brant-Zawadzki M, Yeates A, Crooks LE, Kaufman Norman L. Magnetic resonance imaging of the spinal cord and canal: potentials and limitations. Amer J Neuroradiol 1984; 5 (1): 9-14.

22. Baudin ME, Couvelard A, Krenning E, Öberg K, Steinmüller T, Anlauf M, Wiedenmann B, Salazar R. ENETS consensus guidelines for the management of patients with liver and other distant metastases from neuroendocrine neoplasms of foregut, midgut, hindgut, and unknown primary. Neuroendocrinology 2012; 95 (2): 157-176.

23. Makhani KL, Zeng L, Lam H, Chow Dennis E. Single fraction conventional external beam radiation therapy for bone metastases: a systematic review of randomised controlled trials. Radiother Oncol 2013; 106 (1): 5-14.

24. Stokkel BMP, Chiti A, Lucignani G, Bajetta E, Pauwels EK, Bombardieri van der Hiel $\mathbf{E}$. Effective treatment of bone metastases from a neuroendocrine tumour of the pancreas with high activities of Indium111-pentetreotide. Eur J Endocrinol 2003; 149 (6): 479-483.

25. Nickeleit SV, Mueller-Brand J, Brunner FP, Maecke HR, Mihatsch Moll MJ. Anew cause of renal thrombotic microangiopathy: yttrium 90-DOTATOC internal radiotherapy. Amer J Kidney Dis 2001; 37 (4): 847-851.

26. Teunissen DJ, Bakker JJ, Kooij WH, de Herder PP, Feelders WW, van Eijck RA, Esser CH, Kam JP, Krenning Kwekkeboom BL. Radiolabeled somatostatin analog (177Lu-DOTA0, Tyr3) octreotate in patients with endocrine gastroenteropancreatic tumors. J Clin Oncol 2005; 23 (12): 2754-2762.

27. Jones ACL, Vora J, Poston GJ, Vinjamuri S, Pritchard DM. Assessment of the efficacy and toxicity of 131I-metaiodobenzylguanidine therapy for metastatic neuroendocrine tumours. Brit J Cancer 2008; 98 (6): 1053-1058.

28. Mignon M. Natural history of neuroendocrine enteropancreatic tumors. Digestion 2000; 62 (Suppl 1): 51-58.

29. Singletary $\mathrm{SE}$ et al. A role for curative surgery in the treatment of selected patients with metastatic breast cancer. Oncologist 2003; 8 (3): 241-251.

Received April 8, 2017. Accepted April 27, 2017. 